\title{
How to weight Hasse matrices and reduce incomparabilities
}

\author{
Francesca Grisoni, Viviana Consonni, Serena Nembri, Roberto Todeschini * \\ Milano Chemometrics and QSAR Research Group, Department of Earth and Environmental Sciences, University of Milano-Bicocca, P.za della Scienza 1, Milano 20126, Italy
}

\section{A R T I C L E I N F O}

\section{Article history:}

Received 29 June 2015

Received in revised form 29 July 2015

Accepted 4 August 2015

Available online 11 August 2015

\section{Keywords:}

Multi-criteria decision making

Hasse diagrams

weighted regularized Hasse

wR-Hasse

\begin{abstract}
A B S T R A C T
This work presents a modified version of Hasse diagram technique, the weighted Regularized Hasse (wR-Hasse), which aims to reduce the number of incomparabilities and derive weighted rankings of the objects.

These objectives are accomplished by (a) introducing a mathematical threshold on the definition of incomparability and (b) weighting criteria according to their relevance.

In order to test the new approach, we used eight data sets from literature, aiming at extensively investigating the effect of thresholds and weighting schemes on the outcome.

Results showed how (a) wR-Hasse effectively reduces the number of incomparabilities with respect to the original Hasse and (b) weighting schemes tune the contribution of relevant criteria to the final outcome. Moreover, this approach allows to obtain statistics useful to further investigate data structure and relationships between object ranks.
\end{abstract}

(C) 2015 Elsevier B.V. All rights reserved.

\section{Introduction}

Hasse diagrams (HD) [1], named after Helmut Hasse, are a useful technique to perform partial order ranking, which consists in defining an order between different alternatives on the basis of their criteria values. Ordering techniques are of great importance for multi-criteria decision-making (MCDM) issues, which aim to rationally identify the best candidate among several alternatives, described by multiple (and often conflicting) criteria [2]. In this context, multi-criteria ordering techniques allow to deal with widely diverse and often very complex decision-making problems.

The rationale of HD ordering is very simple: when one alternative has better (or equal) values for all its criteria than another one, the two alternatives are comparable and an order can be set. On the contrary, if at least one pair of criteria is in conflict, the objects are incomparable and the ordering is not possible. In this way, partial ordering techniques differ from total ordering techniques (e.g., simple average ranking [3], Copeland score [4], and weighted Power-Weakness Ratio [5]), which always set an ordering. Once a partial order is established, relations between alternatives are represented as graphs in the socalled Hasse diagrams, in which elements are vertices and ordering relationships are edges. The potential of HD approach lays in its ability of logically sorting objects and giving them a structure. For this reason, HD find successful application to MCDM purposes in many fields, such

\footnotetext{
* Corresponding author at: Department of Earth and Environmental Sciences, University of Milano-Bicocca, P.za della Scienza 1, Milano 20126, Italy.

E-mail address: roberto.todeschini@unimib.it (R. Todeschini).
}

as chemistry and environmental sciences [6], biotechnology [7], computer [8], and social sciences [9]. However, an Achilles' heel of HD is toward data sets with many criteria, where often a large number of incomparabilities are observed [10]. Such cases lead to a less effective representation and often to no ordering at all. Moreover, Hasse theory considers all criteria as equally relevant in determining the final data structure. However, in data analysis and MCDM issues, criteria not always have the same relevance for the problem under analysis, and the possibility to give more relevance to some of them allows for a more feasible and rational application of the technique. Several solutions to these issues have been proposed, such as the iterative application of fuzzy methods and Monte Carlo simulations to reduce the number of incomparabilities ([11, 12]), or the creation of weighted aggregated indicators $([13,14])$.

In the present work, we propose a novel approach, the weighted regularized Hasse (wR-Hasse), which aims (a) to smoothen the number of incomparabilities through a mathematical threshold acting on the definition of incomparability and (b) to weight the criteria according to previous knowledge. This approach is based on a simple algebraic data transformation and allows (a) to obtain more interpretable Hasse diagrams, by reducing the number of incomparabilities, (b) to give more importance to relevant criteria, and (c) to obtain statistics about the objects ordering from a family of weighted regularized Hasse matrices.

After introducing wR-Hasse theory, we use eight literature-based MCDM data sets to discuss in depth its ability to reduce incomparabilities, the effect of weighting schemes on the outcome, and how the statistics help gathering insights about data structure and object ranks. 


\section{Theory}

\subsection{Weighted regularized Hasse theory}

Let $\mathbf{X}$ be a data matrix comprising $n$ objects described by $p$ criteria. A weighted count matrix $\left(\mathbf{T}^{\mathrm{W}}\right)$ can be obtained by defining its elements $\left(t_{i j}^{\mathrm{W}}\right)$ as follows:

$t_{i j}^{\mathrm{W}}=\sum_{k=1}^{p} w_{k} \cdot \delta_{i j, k} \quad$ where $\quad \delta_{i j, k}=\left\{\begin{array}{cl}1 & \text { if } x_{i k} \triangleright x_{j k} \\ 0.5 & \text { if } x_{i k} \triangleq x_{j k} \\ 0 & \text { if } x_{i k} \triangleleft x_{j k}\end{array} \quad\right.$ and $\quad \sum_{k=1}^{p} w_{k}=1$

where $w_{k}$ is the weight given to the $k$ th criterion and $x_{i k}$ is the value of the $k$ th criterion for the $i$ th object.

In other words, for each $k$ criterion $(1 \leq k \leq p)$, each ith element will dominate against the $j$ th if its value is better than that of $j$ (i.e., $x_{i k} \triangleright x_{j k}$ ). If the contrary happens $\left(x_{i k} \triangleleft x_{j k}\right)$, the $j$ th element will dominate over $i$. Finally, if the values are equal $\left(x_{i k} \triangleq x_{j k}\right)$, the two objects "tie the comparison" for the $k$ th criterion and half a credit is given to both. This weighting scheme was already introduced in our previous work [5], as it is a very simple and efficient way to weight criteria and compare objects. Note that all the values of $\mathbf{T}^{\mathrm{W}}$ range from 0 to 1 and $t_{i j}^{\mathrm{W}}+t_{j i}^{\mathrm{W}}=1$.

The transition from $\mathbf{T}^{\mathrm{W}}$ to a weighted regularized Hasse matrix $\left(\mathbf{H}^{\mathrm{R}}\right)$ is obtained by introducing a mathematical threshold on the definition of incomparability. In particular, by means of a threshold $t^{*}$, a weighted regularized Hasse matrix $\mathbf{H}^{\mathrm{R}}\left(t^{*}\right)$ can be obtained as follows:

$\left[\mathbf{H}^{\mathrm{R}}\left(t^{*}\right)\right]_{i j}=\left\{\begin{array}{rl}+1 & \text { if } t_{i j}^{\mathrm{W}} \geq t^{*} \\ -1 & \text { if } t_{i j}^{\mathrm{W}} \leq 1-t^{*} \\ 0 & \text { otherwise }\end{array} \quad 0.55 \leq t^{*} \leq 1\right.$

where $t^{*}$ can be chosen in the range of 0.55 and 1 . The meaning of $t^{*}$ is very intuitive: it corresponds to the fraction of criteria for which an object $j$ has to be better than an object $i$ (or vice versa) in order to set an ordering between the two. For instance, when $t^{*}=0.75$ and the criteria are equally weighted, $i$ dominates over $j$ if it has better values for the $75 \%$ of the criteria. When a different weighting scheme is adopted, $i$ dominates over $j$ when the weights of the criteria for which $i$ is better than $j$ sum up to 0.75 .

In case of no draws, $t^{*}=1$ provides the original Hasse matrix, while in case of some draws, a more restrictive Hasse matrix is obtained. In fact, according to $\mathrm{wR}$-Hasse approach, when $t^{*}=1, i$ has to be better than $j$ for all its criteria (or vice versa) in order set an ordering between the two, while original Hasse also allows some case of draws.

By progressively decreasing $t^{*}$, i.e., by loosening up the definition of incomparability, also the number of incomparabilities tends to decrease. In this way, the efficiency of HD technique can be tuned by varying $t^{*}$, moving to the most restrictive case $\left(t^{*}=1\right)$ to cases where the number of incomparabilities is significantly reduced.

Seldom, it could happen that the number of incomparabilities is constant above the whole range of $t^{*}$, for what we here define as strong

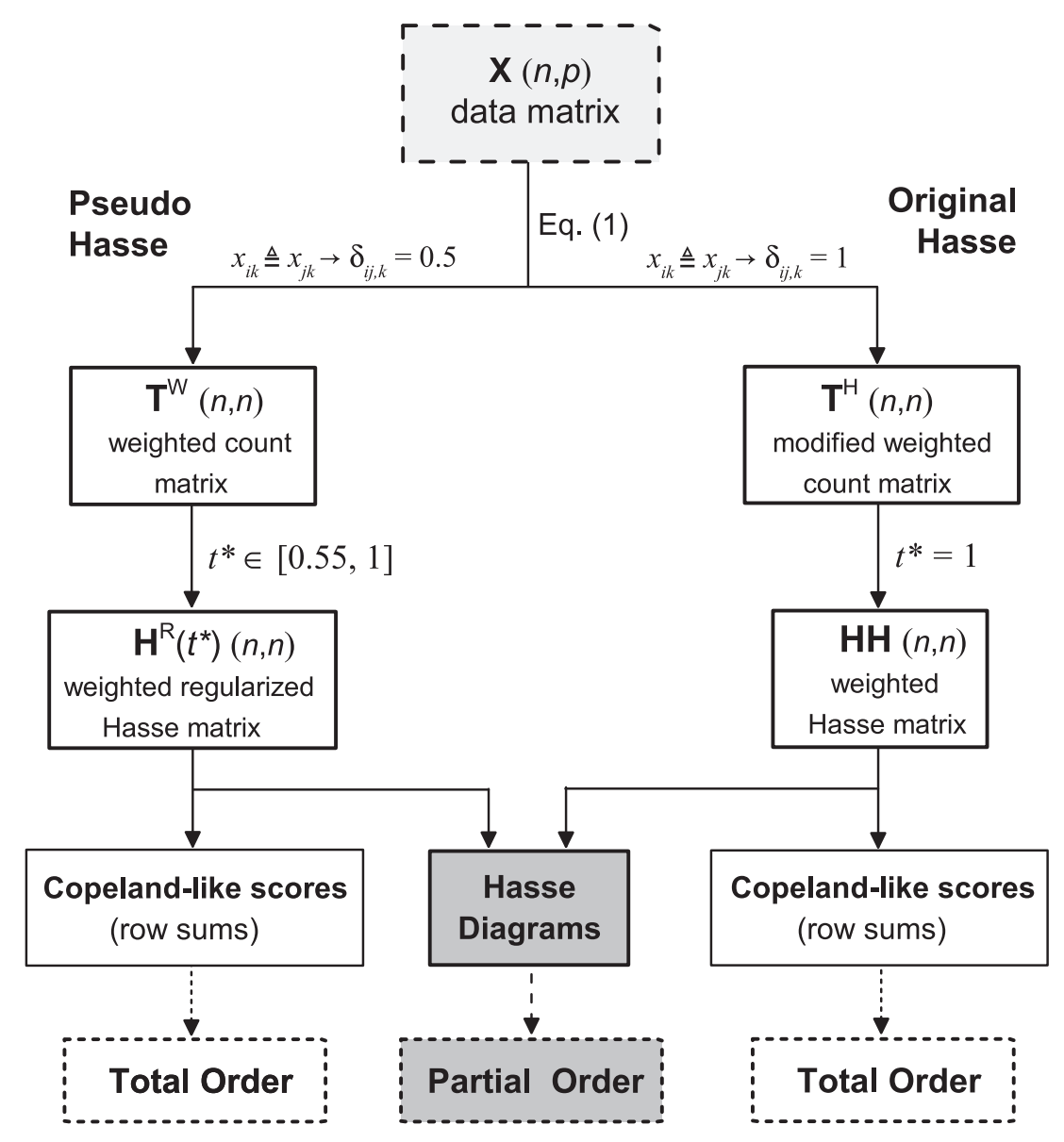

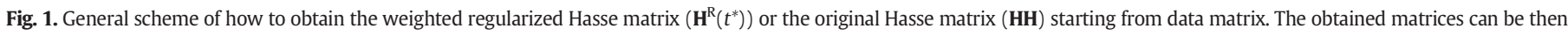
used to draw Hasse diagrams and obtain partial orders or to obtain total orders through the Copeland-like scores (Eq. (3)). 
irreducibility. More often, the number of incomparabilities decreases when decreasing $t^{*}$ but without reaching zero. In this case, we can speak of weak irreducibility.

The set of all possible $t^{*}$ values allows to obtain a family of wRHasse matrices $\left(\mathbf{H}^{\mathrm{R}}\right)$, whose cardinality depends on the number of criteria and the chosen set of weights. The total number $(T)$ of weighted regularized Hasse matrices can be obtained a posteriori directly from $\mathbf{T}^{\mathrm{W}}$ (rounded to the second decimal place) after analysing how many different values greater to or equal than 0.55 are present; these values constitute the optimal set of thresholds. By varying $t^{*}$ across the whole set, one can also obtain statistics that reflect the relevance of the each object ordering and gain information about data structure. This will be discussed in details in the next Section 2.2.
From each member of the $\mathbf{H}^{\mathrm{R}}$ family, also a total ordering can be obtained. In particular, one can calculate scores that are conceptually similar to those of the Copeland method [4], which compares the alternatives in a pairwise manner and sorts them according to their number of victories minus the number of defeats against the remaining ones. In our case, Copeland-like scores can be obtained as matrix row sums:

$C_{i}\left[\mathbf{H}^{\mathrm{R}}\left(t^{*}\right)\right]=\sum_{j=1}^{n}\left[\mathbf{H}^{\mathrm{R}}\left(t^{*}\right)\right]_{i j}$

where $C_{i}$ is the Copeland-like score of the $i$ th object obtained from the $\mathbf{H}^{\mathrm{R}}\left(t^{*}\right)$ matrix with the chosen $t^{*}$ threshold. This score, as the original

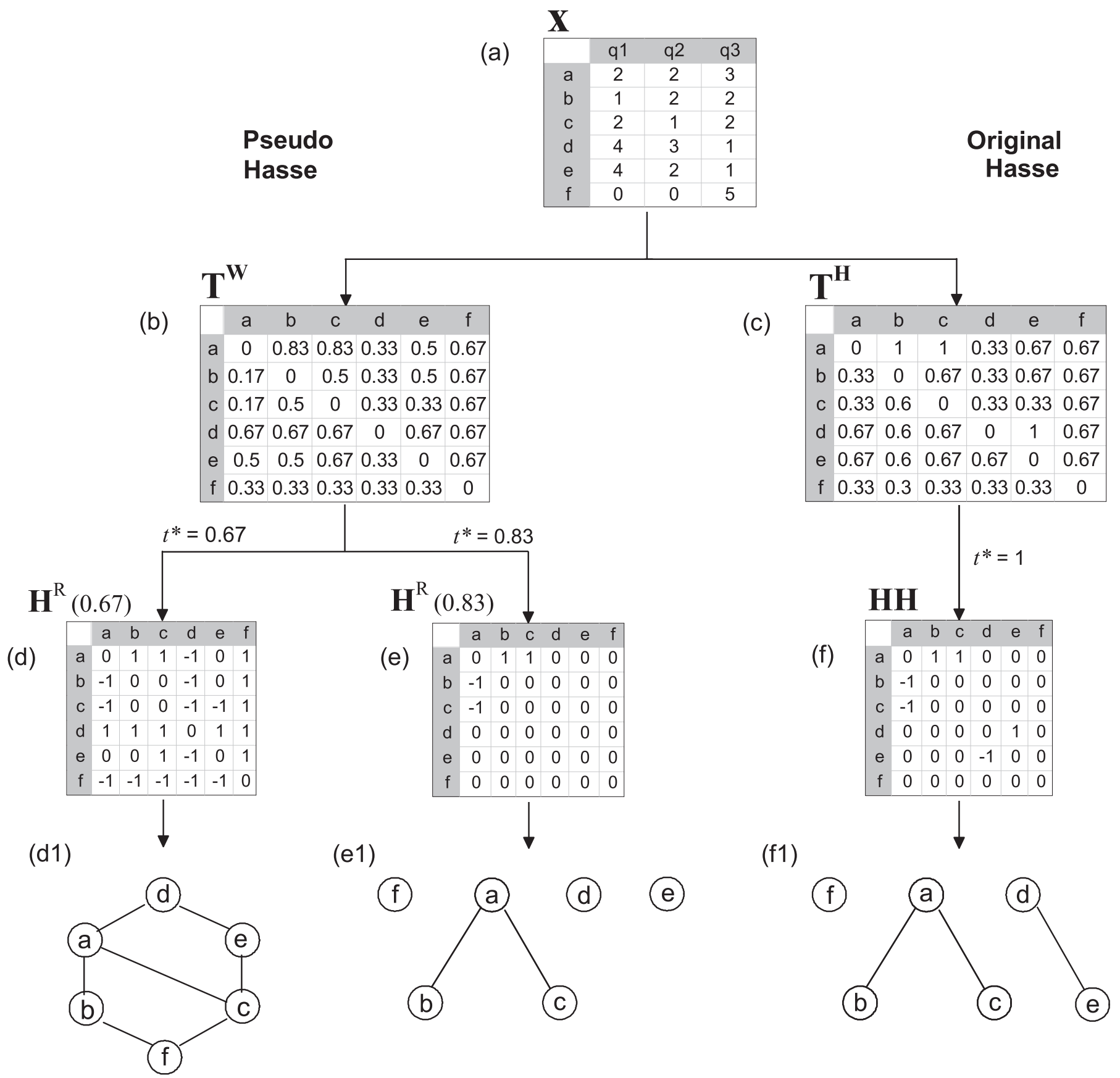

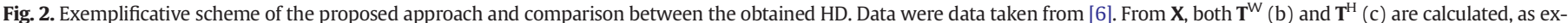

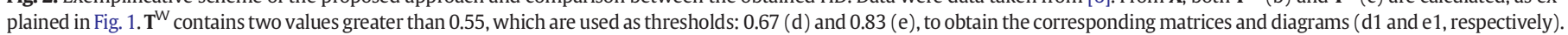
From $\mathbf{T}^{\mathrm{H}}$, the original Hasse matrix can be obtained with $t^{*}=1$ (f) with the corresponding Hasse diagram (f1). 


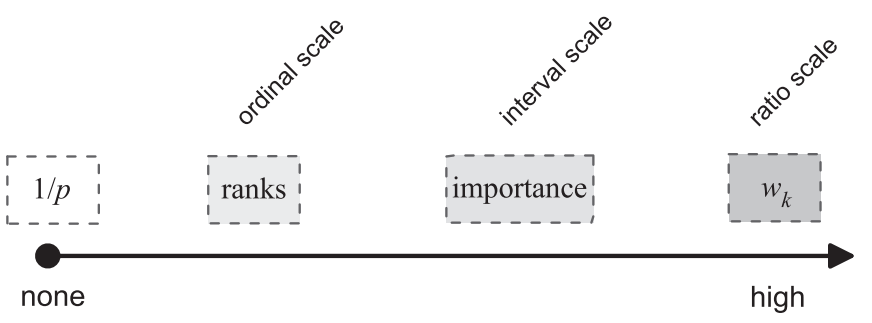

a-priori knowledge

Fig. 3. Different weighting schemes according to the degree of knowledge about the problem.

Copeland does, ranks objects according to their proportion of pairwise victories and defeats against the remaining ones for all the criteria. A conceptual flow diagram can be found in Fig. 1, which also shows how the original Hasse matrix $(\mathbf{H H})$ is obtained through a modified weighted count matrix $\mathbf{T}^{\mathrm{H}}$, assigning a full credit instead of half a credit to the equality condition of Eq. (1) (i.e., $x_{i k} \triangleq x_{j k} \rightarrow \delta_{i j, k}=1$ ).

In order to better clarify the procedure, a simple example with 6 objects and 3 criteria is discussed in Fig. 2; for the sake of simplicity, all the criteria were equally weighted (i.e., $w_{j}=1 / p=0.33$ ).

Fig. 2 shows how, from $\mathbf{X}$, the weighted count matrix ( $\mathbf{T}^{\mathrm{w}}$ ) (Fig. 2b) and the modified weighted count matrix $\mathbf{T}^{\mathrm{H}}$ (Fig. 2c) are calculated. In this case, $\mathrm{T}^{\mathrm{W}}$ contains only two values greater than $0.55(0.67,0.83)$, which are used to obtain a two-membered $\mathbf{H}^{\mathrm{R}}$ family $(\mathrm{d}, \mathrm{e})$ with the corresponding diagrams (d1, e1). Similarly, from $\mathbf{T}^{\mathrm{H}}$, the corresponding original Hasse matrix $\mathbf{H H}\left(t^{*}=1\right)$ and diagram are obtained (f, f1). It can be noted that the maximum possible threshold $t^{*}=0.83$ leads to a larger number of incomparabilities than the original Hasse diagram (f1). In fact, in the original Hasse approach, the objects e and $d$ are comparable, since draws are allowed to set an ordering between object when the remaining criteria are not in conflict. On the contrary, in the wR-Hasse approach, the draws only score half a credit, resulting in $t^{\mathrm{W}}=0.63$ and leading to an incomparability for $t^{*}=0.83$. This situation changes for $t^{*}=0.67$, which leads to a significant reduction of the number of incomparabilities with respect to the original Hasse diagram (see d1 versus f1).

\subsection{Statistics from weighted regularized Hasse family}

As already mentioned, from each $\mathbf{H}^{\mathrm{R}}\left(t^{*}\right)$, a Copeland-like score can be obtained for each object along with the corresponding ranks. This corresponds to a set of ranks for the $\mathbf{H}^{\mathrm{R}}$ family, from which useful statistics can be derived, such as minimum/maximum ranks, arithmetic mean, and standard deviations. Arithmetic mean gives the average ranking of objects, while the standard deviation gives the ranking variability across the set of $t^{*}$. For instance, objects that are ranked always to the same position independently from $t^{*}$ (e.g., invariant to the regularization procedure) will have a standard deviation equal to zero. On the contrary, those having a large standard deviation will be characterized by high rank variability within the $\mathbf{H}^{\mathrm{R}}$ family. Moreover, the coefficient of variation (CV\%) can be also calculated in order to obtain a measure independent of the average rank.

Table 1

Weights given to 5 criteria when their rankings and $\alpha$ parameter vary (Eq. (5)).

\begin{tabular}{llllll}
\hline Criterion & 1 & 2 & 3 & 4 & 5 \\
\hline$\alpha \backslash$ Rank & 1 & 2 & 3.5 & 3.5 & 5 \\
0.5 & 0.310 & 0.219 & 0.166 & 0.166 & 0.139 \\
1.0 & 0.440 & 0.220 & 0.126 & 0.126 & 0.088 \\
1.5 & 0.572 & 0.202 & 0.087 & 0.087 & 0.051 \\
2.0 & 0.688 & 0.172 & 0.056 & 0.056 & 0.028 \\
\hline
\end{tabular}

Table 2

Weights given to 5 criteria according to different vectors of relative importance $(v)$.

\begin{tabular}{|c|c|c|c|c|c|}
\hline \multirow[b]{2}{*}{$v$} & \multicolumn{5}{|c|}{ Criteria } \\
\hline & 1 & 2 & 3 & 4 & 5 \\
\hline 21111 & 0.333 & 0.167 & 0.167 & 0.167 & 0.167 \\
\hline 31111 & 0.429 & 0.143 & 0.143 & 0.143 & 0.143 \\
\hline 33311 & 0.273 & 0.273 & 0.273 & 0.091 & 0.091 \\
\hline 53331 & 0.333 & 0.200 & 0.200 & 0.200 & 0.067 \\
\hline 55221 & 0.333 & 0.333 & 0.133 & 0.133 & 0.067 \\
\hline
\end{tabular}

\subsection{Choosing weights}

Criteria weighting is often perceived as an arbitrary decision and it can also become a source of controversy when weights have to be chosen by a group of decision makers. However, the possibility to weight criteria (when transparent and based on knowledge about the problem), is an opportunity that should not be neglected.

On the basis of the knowledge about the problem, different approaches can be used to define weights (Fig. 3):

1. Criteria can be weighted according to their relevance $\left(r_{j}\right)$ (moderate knowledge). A method for this calculation has been already proposed [16], as the following:

$$
w_{j}(\alpha)=\frac{Q / r_{j}^{\alpha}}{\sum_{j=1}^{p} Q / r_{j}^{\alpha}}
$$

where $r_{j}^{\alpha}=\exp \left[\alpha \cdot \ln \left(r_{j}\right)\right]$ and $Q=\prod_{j=1}^{p} r_{j}^{\alpha} \cdot w_{j}(\alpha)$ is the normalized weight for the $j$ th criterion, and $\alpha$ is a smoothing parameter, which influences the differences between the weights of highranked criteria and those of low-ranked ones. In particular, when $\alpha=0$, criteria are equally weighted, i.e., they are independent of their ranks, while when $\alpha$ increases, also the differences between weights increases (Table 1 ).

2. By assigning a relative importance $\left(v_{\mathrm{j}}\right)$ to the criteria, the greater the importance, the larger the corresponding weight (moderate-to-high knowledge). The weight $w_{j}$ is then obtained by dividing $v_{\mathrm{j}}$ by the sum of all the $p$ importance values (Table 2); Eq. (5) can be also used, by replacing $r_{i}$ with $1 / v_{\mathrm{j}}$.

3. By directly assigning weights, when the degree of knowledge is very high.

4. When no a priori knowledge is available, the $p$ criteria should be equally weighted as $1 / p$.

Moreover, in ref. [16], it was observed how for some ranges of weights, the same rankings/diagrams are obtained. Therefore, the discussion should only regard those value regions where small changes of the assigned weights can lead to very different outcomes.

Table 3

Data sets under analysis. ID, criteria type, number of objects $(n)$, and criteria $(p)$ are reported.

\begin{tabular}{llllll}
\hline Data set & ID & $n$ & $p$ & Criteria type & Reference \\
\hline Perfluoroalkyl acids & PFA & 7 & 10 & Bioaccumulation properties & {$[17]$} \\
Benzamide derivatives & Benz & 72 & 3 & Receptor inhibition & {$[18]$} \\
Anilines & Anil & 45 & 4 & $\begin{array}{l}\text { Environmental impact } \\
\text { properties }\end{array}$ & {$[19]$} \\
& & & & Algal toxicity & {$[20]$} \\
Pesticides & Pest & 17 & 6 & Ald & {$[21]$} \\
Bibliometric index & BI & 26 & 27 & Bibliometric indices & {$[22]$} \\
$\begin{array}{l}\text { Analytical chemistry } \\
\text { journals }\end{array}$ & JAC & 86 & 9 & Journal metrics & \\
$\begin{array}{l}\text { Classification methods } \\
\text { Cities }\end{array}$ & CMeth & 10 & 32 & Non-error rate (NER) & {$[23]$} \\
\hline
\end{tabular}


Table 4

$\mathrm{H}^{\mathrm{R}}$ families for CMeth, JAC, Pest and Benz data sets; $\mathrm{HH}$ is the original Hasse matrix. Threshold values $\left(t^{*}\right)$, the percentage of incomparabilities (IT\%), and the percentage of objects with at least one incomparability (IO\%) are also reported.

\begin{tabular}{|c|c|c|c|c|c|c|c|}
\hline $\mathrm{H}$ family & $t^{*}$ & IT\% & $\mathrm{IO} \%$ & $\mathrm{H}$ family & $t^{*}$ & IT\% & $\mathrm{IO} \%$ \\
\hline \multicolumn{4}{|l|}{ CMeth } & \multicolumn{4}{|l|}{ JAC } \\
\hline $\mathrm{H}^{1}$ & 0.55 & 13.3 & 70.0 & $\mathrm{H}^{1}$ & 0.56 & 0.2 & 20.9 \\
\hline $\mathrm{H}^{2}$ & 0.56 & 20.0 & 80.0 & $\mathrm{H}^{2}$ & 0.61 & 13.7 & 98.8 \\
\hline $\mathrm{H}^{3}$ & 0.58 & 22.2 & 80.0 & $\mathrm{H}^{3}$ & 0.67 & 14.0 & 98.8 \\
\hline $\mathrm{H}^{4}$ & 0.59 & 31.1 & 90.0 & $\mathrm{H}^{4}$ & 0.72 & 30.0 & 100.0 \\
\hline $\mathrm{H}^{5}$ & 0.61 & 37.8 & 100.0 & $\mathrm{H}^{5}$ & 0.78 & 30.4 & 100.0 \\
\hline $\mathrm{H}^{6}$ & 0.63 & 40.0 & 100.0 & $\mathrm{H}^{6}$ & 0.83 & 41.4 & 100.0 \\
\hline $\mathrm{H}^{7}$ & 0.64 & 44.4 & 100.0 & $\mathrm{H}^{7}$ & 0.89 & 41.7 & 100.0 \\
\hline $\mathrm{H}^{8}$ & 0.67 & 51.1 & 100.0 & $\mathrm{H}^{8}$ & 0.94 & 63.1 & 100.0 \\
\hline $\mathrm{H}^{9}$ & 0.69 & 60.0 & 100.0 & $\mathrm{H}^{9}$ & 1.00 & 63.3 & 100.0 \\
\hline $\mathrm{H}^{10}$ & 0.72 & 62.2 & 100.0 & $\mathrm{HH}$ & 1.00 & 63.1 & 100.0 \\
\hline $\mathrm{H}^{11}$ & 0.73 & 68.9 & 100.0 & \multicolumn{4}{|l|}{ Pest } \\
\hline $\mathrm{H}^{12}$ & 0.75 & 73.3 & 100.0 & $\overline{\mathrm{H}^{1}}$ & 0.67 & 2.9 & 29.4 \\
\hline $\mathrm{H}^{13}$ & 0.80 & 75.6 & 100.0 & $\mathrm{H}^{2}$ & 0.83 & 8.1 & 64.7 \\
\hline $\mathrm{H}^{14}$ & 0.81 & 82.2 & 100.0 & $\mathrm{H}^{3}$ & 1.00 & 11.0 & 64.7 \\
\hline $\mathrm{H}^{15}$ & 0.83 & 86.7 & 100.0 & $\mathrm{HH}$ & 1.00 & 11.0 & 64.7 \\
\hline $\mathrm{H}^{16}$ & 0.86 & 88.9 & 100.0 & \multicolumn{4}{|l|}{ Benz } \\
\hline $\mathrm{H}^{17}$ & 0.89 & 91.1 & 100.0 & $\mathrm{H}^{1}$ & 0.67 & 0.7 & 31.9 \\
\hline $\mathrm{H}^{18}$ & 0.92 & 95.6 & 100.0 & $\mathrm{H}^{2}$ & 0.83 & 54.0 & 100.0 \\
\hline \multirow[t]{2}{*}{$\mathrm{HH}$} & 1.00 & 100.0 & 100.0 & $\mathrm{H}^{3}$ & 1.00 & 55.0 & 100.0 \\
\hline & & & & $\mathrm{HH}$ & 1.00 & 54.2 & 100.0 \\
\hline
\end{tabular}

\section{Data sets}

In order to test wR-Hasse approach, eight data sets of different type were used (Table 3): four of chemical and environmental interest (PFA, Benz, Anil, Pest), two regarding scientific research evaluation (BI and JAC), and two about comparison of classification methods and cities (CMeth and Cit, respectively). Data sets are entirely provided as Supplementary Material of this work.

(a1)
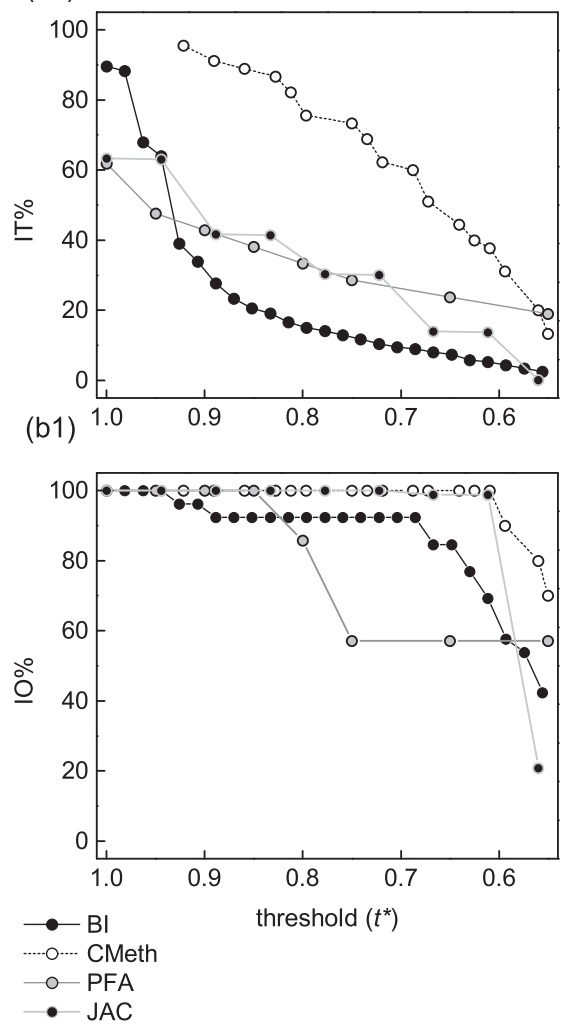

\section{Results and discussion}

\subsection{Incomparability analysis}

For each data set, we obtained the family of $T$ weighted regularized Hasse matrices, as explained above. In addition, also the original Hasse matrix (HH) was considered. For each of these matrices, we calculated (a) the percentage of incomparabilities (IT\%.), i.e., the ratio of the number of incomparabilities over the theoretical maximum $(n \cdot(n-1) / 2)$ and (b) the percentage of objects having at least one incomparability (IO\%). Note that, when the greatest $t^{*}$ value is equal to 1 , IT $\%(\mathbf{H H}) \leq \mathrm{IT} \%\left(\mathbf{H}^{\mathrm{R}}(1)\right)$. In Table 4 , the obtained $\mathbf{H}^{\mathrm{R}}$ families for CMeth, JAC, Pest, and Benz are shown. Fig. 3 depicts the graphical trends of IT\% (Fig. 4a) and IO\% (Fig. 4b) for all data sets when $t^{*}$ is varied.

From Fig. 4, the effect of reduction of incomparabilities when decreasing $t^{*}$ values is visible, with different trends for each data set. The percentage of incomparabilities (IT\%) decreases monotonically, often sharply, while, as expected, IO\% decreases in a less pronounced way. In this last case, for some data sets, percentages of objects having at least one incomparability with one or more objects still remain high for low $t^{*}$ values.

The data set CMeth, which has the biggest variation of IO\% within the family, was used as example to graphically show the effect of the threshold on wR-Hasse diagrams and number of incomparabilities (Fig. 5). In this case, the original Hasse diagram (not reported) would lead to all objects being incomparable. This is one of those cases where the large number of criteria (32) reflects in a high degree of incomparability. One can see how data structure progressively rearranges, with some objects that always occupy similar positions in the orderings (e.g., 10, 7). Note, for example, how objects 5 and 7 are incomparable for $t^{*} \geq 0.73$, but when $t^{*}$ decreases, the ordering is set $(5 \rightarrow 7)$ and remains stable.

(a2)

(b2)
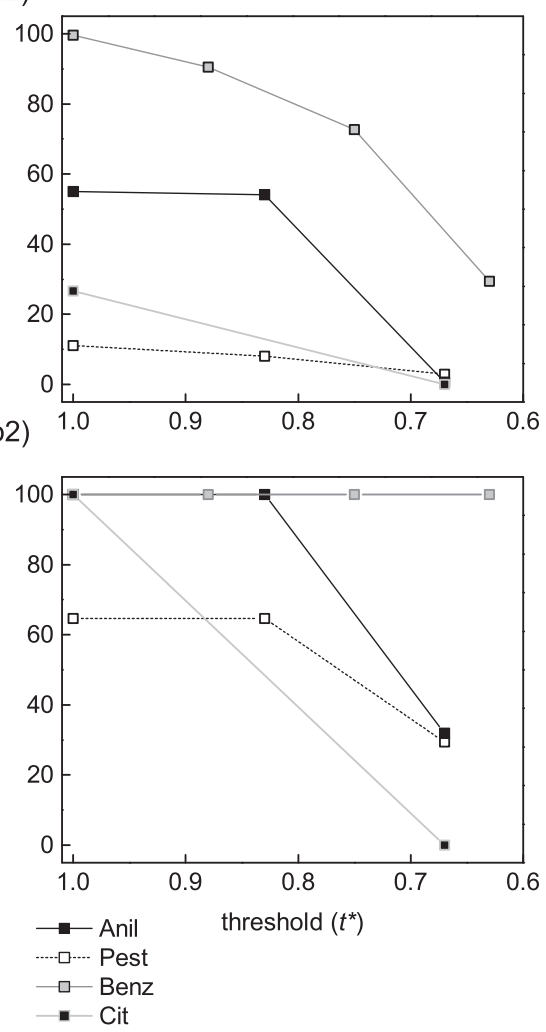

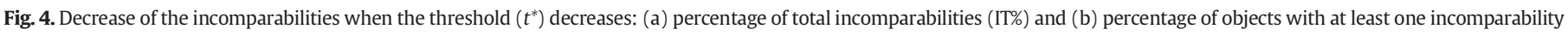
(IO\%). Data sets with more than 4 values of $t^{*}$ are depicted on the left $(\mathrm{a} 1, \mathrm{~b} 1)$ while the others on the right $(\mathrm{a} 2, \mathrm{~b} 2)$ 


$$
t^{*}=0.92
$$

(1)

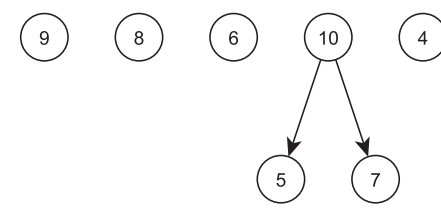

$$
t^{*}=0.73
$$

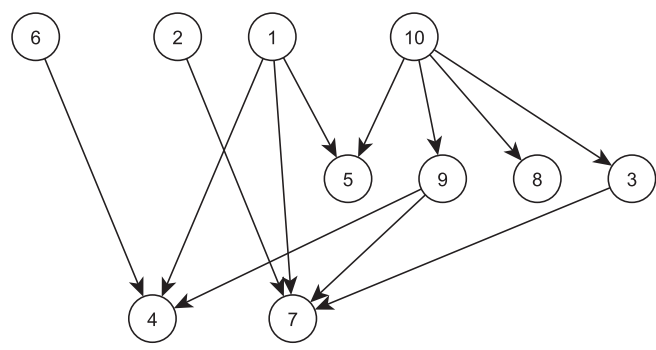

$$
t^{*}=0.58
$$

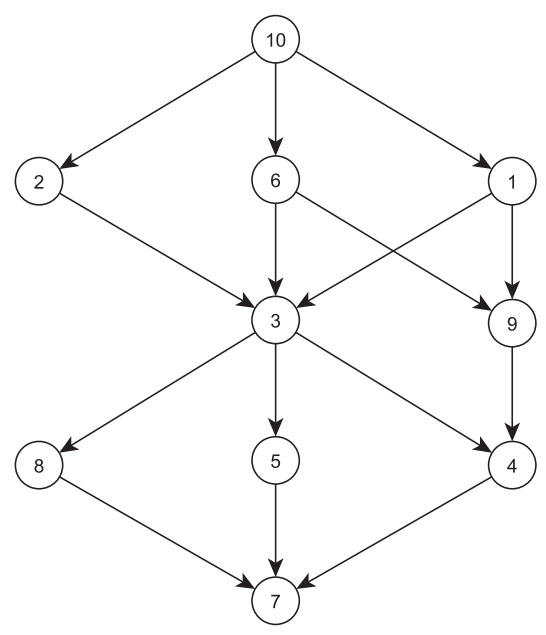

$t^{*}=0.81$

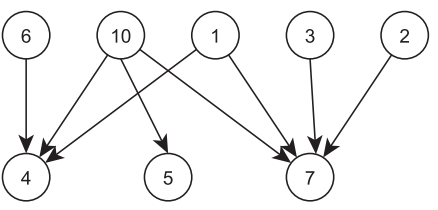

(8)

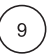

$t^{*}=0.64$

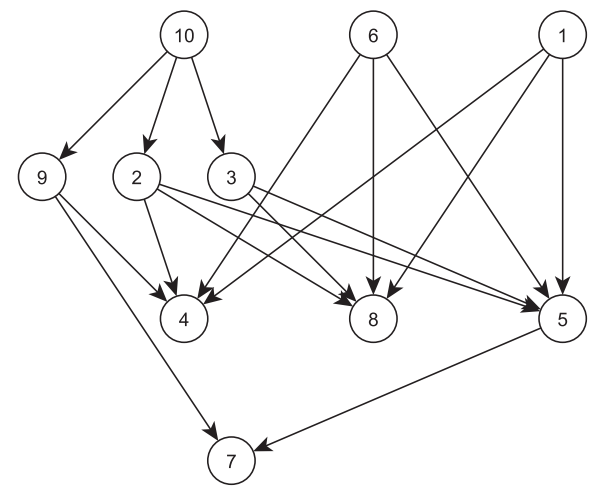

$$
t^{*}=0.55
$$

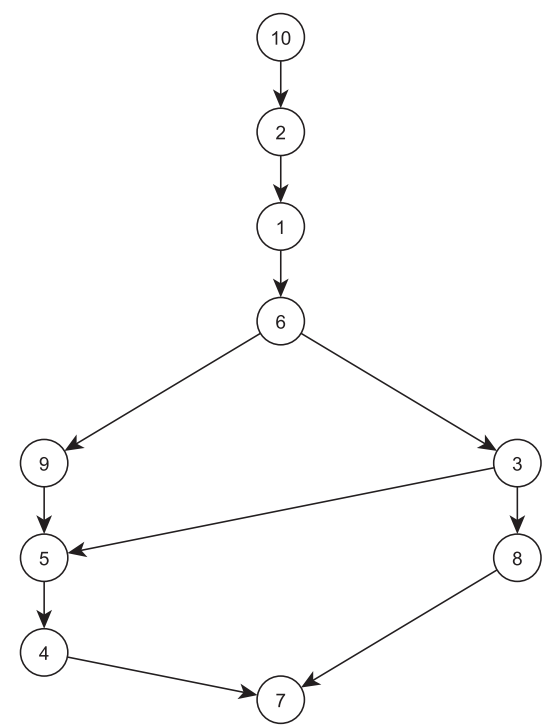

Fig. 5. Different wR-Hasse diagrams for CMeth data set, obtained by six threshold ( $\left.t^{*}\right)$ values. Original Hasse diagram (not reported) has $100 \%$ of incomparable objects.

\subsection{Statistics and multivariate analysis}

Once the set of $T$ thresholds and the corresponding $\mathbf{H}^{\mathrm{R}}$ family are obtained, one has the opportunity to gather some statistical insights about objects ranks across the range of $t^{*}$ values. In this work, in particular, for each data set, we calculated the ranks from Copeland-like scores of (a) all the matrices of the $\mathbf{H}^{\mathrm{R}}$ family, (b) the original Hasse (HH), and (c) the weighted count matrix $\mathrm{T}^{\mathrm{W}}$ (Cop). For the sake of comparison, also wPWR ranks were taken into account since they derive from an eigenvector-eigenvalue decomposition of $\mathbf{T}^{\mathrm{W}}$ [5]. In this way, for each data set, each of the $n$ objects was described by $T+3$ ranks obtained by the approaches listed above, where $T$ is the number of the $\mathbf{H}^{\mathrm{R}}$ family.
We firstly calculated the Kendall concordance (W) [25] for all data sets, which is the appropriate univariate measure for concordance between rankings. The largest $\mathrm{W}$, the greater the concordance. One can note that while the ranks of Pest, Benz, and Cities data sets are almost invariant to the approach used, those of Anil are strongly dependent on the ranking approach $(\mathrm{W}=0.715)$. The other data sets show intermediate behaviour (Table 5).

A multivariate exploration of ranks was also performed using a Principal Component Analysis (PCA). In all cases analysed, the first Principal Component (PC1) explains the average ranking of each object obtained by all the approaches, while the second (PC2) highlights objects whose rankings vary among the approaches. The 
Table 5

Kendall W concordance, percentage of explained variance of PC1, PC2, and complementary explained variance of PC1 (100-PC1).

\begin{tabular}{lcccc}
\hline \multirow{2}{*}{ Data set } & W & \multicolumn{3}{c}{ Explained variance \% } \\
\cline { 3 - 5 } & & PC1 & PC2 & $100-$ PC1 \\
\hline Anil & 0.715 & 80.19 & 12.40 & 19.81 \\
Pest & 0.994 & 99.87 & 0.10 & 0.13 \\
JAC & 0.961 & 96.16 & 3.31 & 3.84 \\
BI & 0.938 & 95.57 & 2.72 & 4.43 \\
CMeth & 0.821 & 93.48 & 3.25 & 6.52 \\
PFA & 0.861 & 93.36 & 2.69 & 6.64 \\
Benz & 0.989 & 98.92 & 0.99 & 1.08 \\
Cities & 0.981 & 98.73 & 1.06 & 1.27 \\
\hline
\end{tabular}

remaining PCs (only explaining a small residual variance) give further information about pairwise comparison between objects. It can be observed that there is a correlation between $\mathrm{W}$ and the variance explained by PC1, since they encode the same type of information (Table 5).

In the next paragraphs, three examples with high, intermediate, and low $\mathrm{W}$ values are used to further discuss wR-Hasse technique. In particular, we chose Pest ( $\mathrm{W}=0.994)$, PFA $(\mathrm{W}=0.861)$ and Anil
$(\mathrm{W}=0.715)$, which are all application of MCDM techniques to environmental risk assessment of chemicals. For each data set, loadings and scores on the first two PCs are depicted in Fig. 6 and results are discussed in depth separately for each of them.

\subsubsection{Pest data set}

Pest data set comprises 17 pesticides described by 6 levels of algal toxicity. This is an example of the advantages offered by MCDM techniques, which allow to account for the whole toxicity profile of chemicals without relying on a single value. The considered statistics (paragraph 2.3) are reported in Table 6. Ranks obtained with wRHasse and total ordering methods (i.e., Cop, PWR) are very similar. This is probably because all criteria have the same meaning and the compounds have coherent trends in their toxicity. This reflects in a low explained variance by PC2 $(0.1 \%)$, while PC1 explains almost the total variance (99.9\%) (Fig. 6a1, b1). PC1 highlights the global ranking: objects with large PC1 scores $(10,7,17,9)$ have the highest average ranks, while those with the smallest PC1 scores (e.g., 12, 2, 8, 4) have the lowest average ranks. As it can be easily noted, all the objects having zero standard deviation $(2-8,10,12,16)$ are perfectly aligned along PC1 with null PC2 scores. These objects are always ranked at the same position by all methods; thus, they can be (a1)

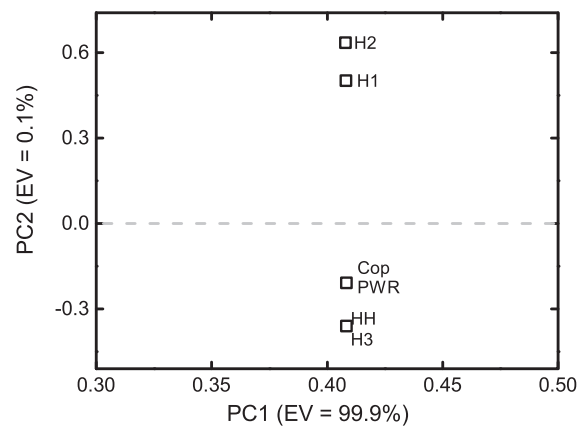

(a2)

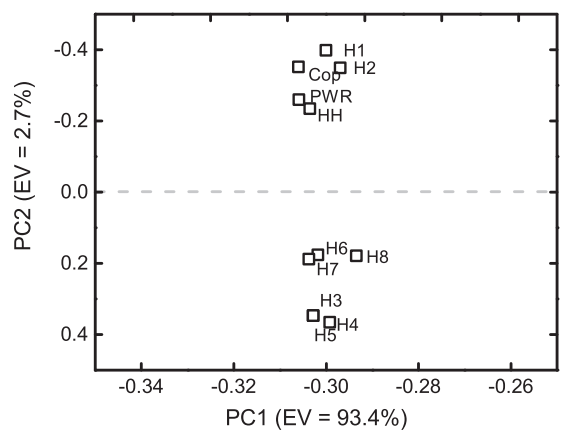

(a3)

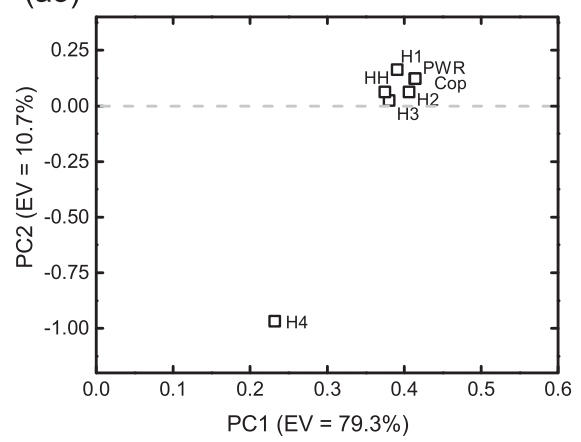

(b1)

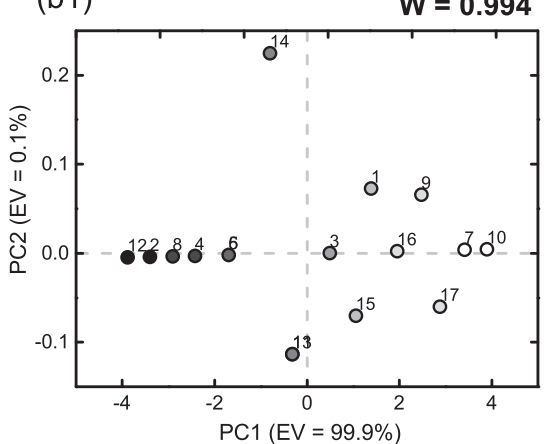

(b2)

$W=0.861$
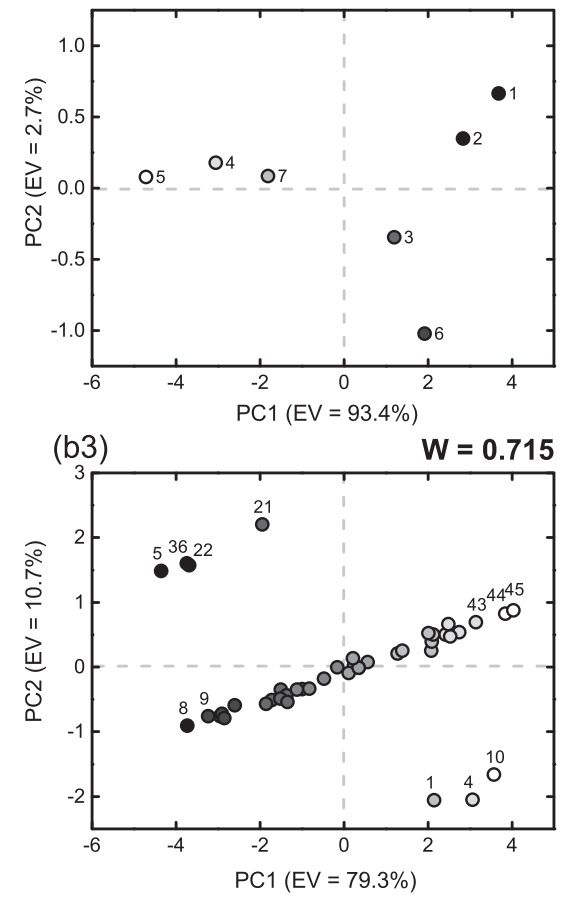

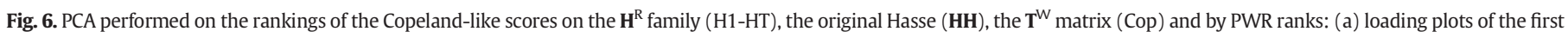

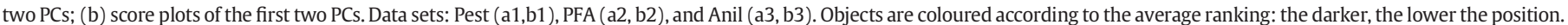




\section{Table 6}

Ranks and summary statistics for the compounds of Pest data set. Ranks correspond to the Copeland-like scores of the $\mathbf{H}^{\mathrm{R}}$ matrices, the original Hasse ( $\mathbf{H H}$ ), the $\mathbf{T}^{\mathrm{W}}$ matrix (Cop), and by PWR ranks.

\begin{tabular}{lrrrrrrrrrrr}
\hline Object & $\mathrm{H}^{1}$ & $\mathrm{H}^{2}$ & $\mathrm{H}^{3}$ & $\mathrm{HH}$ & Cop & PWR & Mean & Std.dev. & CV\% & min & max \\
\hline 1 & 12 & 12 & 11.5 & 11.5 & 12 & 12 & 11.8 & 0.29 & 2.4 & 11.5 & 12 \\
2 & 2 & 2 & 2 & 2 & 2 & 2 & 2.0 & 0.00 & 0.0 & 2 & 2 \\
3 & 10 & 10 & 10 & 10 & 10 & 10 & 10.0 & 0.00 & 0.0 & 10 & 10 \\
4 & 4 & 4 & 4 & 4 & 4 & 4 & 4.0 & 0.00 & 0.0 & 4 & 4 \\
5 & 5.5 & 5.5 & 5.5 & 5.5 & 5.5 & 5.5 & 5.5 & 0.00 & 0.0 & 5.5 & 5.5 \\
6 & 5.5 & 5.5 & 5.5 & 5.5 & 5.5 & 5.5 & 5.5 & 0.00 & 0.0 & 5.5 & 5.5 \\
7 & 16 & 16 & 16 & 16 & 16 & 16 & 16.0 & 0.00 & 0.0 & 16 & 16 \\
8 & 3 & 3 & 3 & 3 & 3 & 3 & 3.0 & 0.00 & 0.0 & 3 & 3 \\
9 & 14 & 14.5 & 14 & 14 & 14 & 14 & 14.2 & 0.29 & 2.0 & 14 & 14.5 \\
10 & 17 & 17 & 17 & 17 & 17 & 17 & 17.0 & 0.00 & 0.0 & 17 & 17 \\
11 & 8 & 8 & 8.5 & 8.5 & 8.5 & 8.5 & 8.2 & 0.29 & 3.5 & 8 & 8.5 \\
12 & 1 & 1 & 1 & 1 & 1 & 1 & 1.0 & 0.00 & 0.0 & 1 & 1 \\
13 & 8 & 8 & 8.5 & 8.5 & 8.5 & 8.5 & 8.2 & 0.29 & 3.5 & 8 & 8.5 \\
14 & 8 & 8 & 7 & 7 & 7 & 7 & 7.7 & 0.58 & 7.5 & 7 & 8 \\
15 & 11 & 11 & 11.5 & 11.5 & 11 & 11 & 11.2 & 0.29 & 2.6 & 11 & 11.5 \\
16 & 13 & 13 & 13 & 13 & 13 & 13 & 13.0 & 0.00 & 0.0 & 13 & 13 \\
17 & 15 & 14.5 & 15 & 15 & 15 & 15 & 14.8 & 0.29 & 1.9 & 14.5 & 15 \\
\hline
\end{tabular}

considered as reference objects, whose ranks do not depend on the approach used. The opposite occurs for object 14, having high PC2 scores: it corresponds to high ranks obtained by $\mathbf{H}^{1}$ and $\mathbf{H}^{2}$ and small by the remaining methods (Fig. 6a1). The opposite happens for objects 11 and 13, which are ranked to lower positions by $\mathbf{H}^{1}-\mathbf{H}^{2}$ and to higher by the remaining methods.

\subsubsection{PFA data set}

A second example of how the analysis of the $\mathbf{H}^{\mathrm{R}}$ family can be used to derive insights about data ordering and structure is given through PFA data set. PFA comprises 7 Perfluorinated acids, a class of emerging contaminants, described by 10 parameters of bioaccumulation in environment. In analogy with Pest, PC1 corresponds to the average rank of objects when all the ranking methods are considered: compound 1 is ranked on average at top positions, while compounds 5, 4, and 7 tend to be ranked at low positions (Fig. 6b2). In this case, the value of $\mathrm{W}$ is smaller than that of Pest and PC2 explains a larger variance (2.7\%). By observing the loading plot (Fig. 6a2), one can notice that all the $\mathbf{H}^{\mathrm{R}}$ matrices obtained with thresholds lower than 0.95 (H3 to H8) have positive loadings on $\mathrm{PC}$, while the remaining methods have negative loadings. This means that $\mathrm{PC} 2$, when comparing the members of the $\mathbf{H}^{\mathrm{R}}$ family, explains the difference between low and high threshold values, corresponding to negative and positive PC2 scores, respectively. Number 5 is on average ranked on the bottom (low PC1 scores) by all the methods (low PC2 scores). In addition, compound n.5 has nearnull PC2 values, meaning that its rank is independent from the method and should be regarded as reliable.

\subsubsection{Anil data set}

The Anil data set comprises 4 indicators of environmental fate and hazard with different meaning, namely, octanol-water partitioning, vapour pressure, biodegradability, and ecotoxicity (predicted no effect concentration). This is probably why, unlike Pest and PFA cases (which comprise very similar criteria), the variability between the obtained ranks is high. This reflects in a variance explained by PC2 (10.7\%) larger than the previous cases. In particular, PC2 mainly explains the difference between $\mathbf{H}^{4}$ (i.e., $t^{*}=1$ ) and the remaining methods (Fig. 6a3, b3). Interestingly, the ranks obtained by $\mathbf{H}^{4}$ classify objects 1,4 , and 10 at the top position, and objects $5,21,22$, and 36 at the last position, while all the remaining compounds are assigned the (same) middle position. This is clearly visible by observing the loading plot (Fig. 6b3). Objects with null or near-null PC1 and PC2 scores (e.g., 30, 25, 39) are ranked at similar positions by all methods and, thus, their rank does not depend from the threshold value chosen.
Table 7

Sets of weights (w1-w25) generated for Anil criteria $\left(C_{1}-C_{4}\right)$.

\begin{tabular}{|c|c|c|c|c|}
\hline \multirow{2}{*}{ ID } & \multicolumn{4}{|c|}{ Weighting scheme } \\
\hline & $C_{1}$ & $\mathrm{C}_{2}$ & $\mathrm{C}_{3}$ & $\mathrm{C}_{4}$ \\
\hline w1 & 0.25 & 0.25 & 0.25 & 0.25 \\
\hline w2 & 0.12 & 0.16 & 0.24 & 0.48 \\
\hline w3 & 0.12 & 0.16 & 0.48 & 0.24 \\
\hline w4 & 0.12 & 0.24 & 0.16 & 0.48 \\
\hline w5 & 0.12 & 0.24 & 0.48 & 0.16 \\
\hline w6 & 0.12 & 0.48 & 0.24 & 0.16 \\
\hline w7 & 0.12 & 0.48 & 0.16 & 0.24 \\
\hline w8 & 0.16 & 0.12 & 0.24 & 0.48 \\
\hline w9 & 0.16 & 0.12 & 0.48 & 0.24 \\
\hline w10 & 0.16 & 0.24 & 0.12 & 0.48 \\
\hline w11 & 0.16 & 0.24 & 0.48 & 0.12 \\
\hline w12 & 0.16 & 0.48 & 0.24 & 0.12 \\
\hline w13 & 0.16 & 0.48 & 0.12 & 0.24 \\
\hline w14 & 0.24 & 0.16 & 0.12 & 0.48 \\
\hline w15 & 0.24 & 0.16 & 0.48 & 0.12 \\
\hline w16 & 0.24 & 0.12 & 0.16 & 0.48 \\
\hline w17 & 0.24 & 0.12 & 0.48 & 0.16 \\
\hline w18 & 0.24 & 0.48 & 0.12 & 0.16 \\
\hline w19 & 0.24 & 0.48 & 0.16 & 0.12 \\
\hline w20 & 0.48 & 0.16 & 0.24 & 0.12 \\
\hline w21 & 0.48 & 0.16 & 0.12 & 0.24 \\
\hline w22 & 0.48 & 0.24 & 0.16 & 0.12 \\
\hline w23 & 0.48 & 0.24 & 0.12 & 0.16 \\
\hline w24 & 0.48 & 0.12 & 0.24 & 0.16 \\
\hline w25 & 0.48 & 0.12 & 0.16 & 0.24 \\
\hline
\end{tabular}

\subsection{Weighting criteria}

In all the previous cases, results were discussed assuming equal weights for all criteria (i.e., $w=1 / p$ ). In order to evaluate the effect of weighting scheme variation on the outcomes, we chose Anil data set, composed by four criteria of different meaning. The set of weights was arbitrarily defined by assigning all the possible combination of ranks to the criteria and then calculating the corresponding weights according to Eq. (5) $(\alpha=1)$. For the sake of comparison, also the case with equal weights $(w=1 / p=0.25$ ) was used (Table 7$)$. For simplicity, we chose $t^{*}=0.75$, a reasonable threshold when 4 criteria are present. For each of the obtained $\mathbf{H}^{\mathrm{R}}$ matrices, Copeland-like scores and corresponding rankings were then calculated, as already explained.

Similarly to the previous examples, a PCA allowed the multivariate analysis of the ranks obtained by each weighting scheme (Fig. 7). Even in this case, PC1 is quantitatively related to the mean rank of each object across the whole set of weighting schemes (Fig. 7a): objects with high PC1 scores are, on average, ranked in last positions, while those with small PC1 scores are ranked at top positions. This allows to immediately identify a global ordering between objects. PC2 and PC3 (explaining the $12.3 \%$ and the $10.0 \%$ of the variance, respectively) are related to the ranking variation when different weighting schemes are adopted. In other words, objects with near-null PC2 and PC3 scores will always be ranked at similar positions, independent of the chosen weighting scheme (e.g., 5, 31, 40, 44, 45), while those far from zero values in at least one of the components 2 and 3 (e.g., 14, 19, 20, 21, 24, 36, 37, 41 ) will be much influenced by criteria weights.

When observing the loading plots (Fig. 7b), one can notice a clustering according to which criterion was assigned the largest weight. For example, when the third criterion (C3) is weighted 0.48 , independent of the other weights, the obtained ranks are all the same. In other cases, e.g., when $\mathrm{C} 2$ is given the largest weight, two clusters can be observed on both the components, according to the high ranks ( 3 or 4 ) given to the remaining criteria.

The previous observations confirm: (1) the effect of the weights on the obtained outcome and (2) how, for some ranges of weights, the same rankings are obtained. This approach allows to have a global 
(a1)

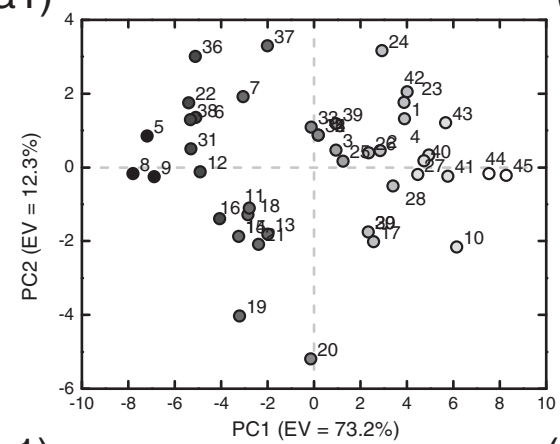

(b1)

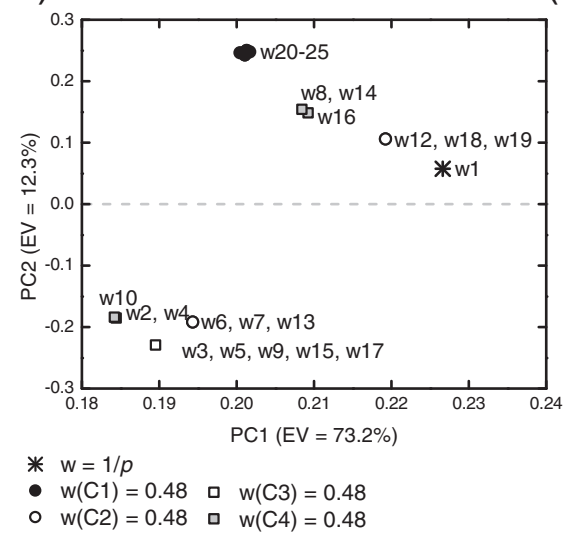

(a2)

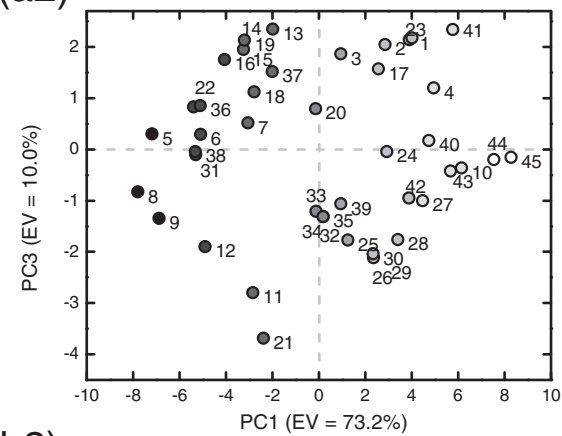

(b2)

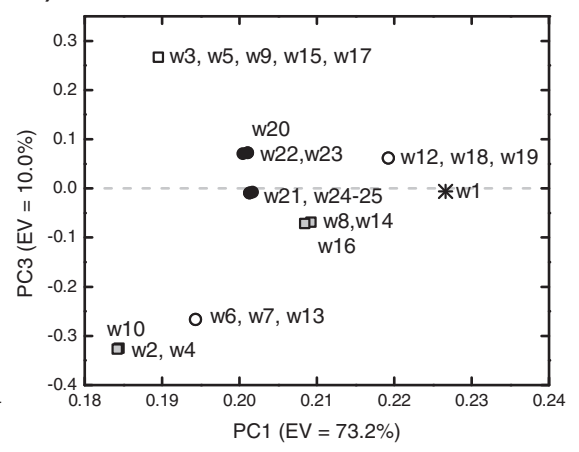

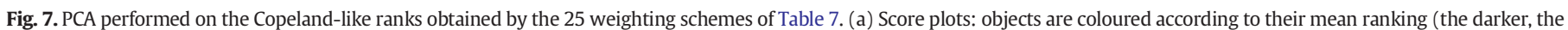
lower). (b) Loading plots: symbols represent the criterion with the highest assigned weight (0.48) and labels represent the IDs of Table 7.

overview of data structure and criterion/weight influence on the outcome and to identify those object having a "robust" ranking, independent of the adopted set of weights. Moreover, the selection of one weighting scheme for each cluster of weights can sensibly reduce the alternatives to analyze and the debate could only regard those weighting schemes which greatly influence the object ranks.

\section{Conclusions}

This paper presented a new technique (wR-Hasse) to weight Hasse diagrams and to reduce the number of incomparabilities, on the basis of the following: (a) a weighting procedure applied to original data and (b) on a threshold to define the incomparability between objects $\left(t^{*}\right)$. To test our new approach, we used 8 data sets taken from literature. This work showed how, by progressively decreasing $t^{*}$, the number of incomparabilities of Hasse diagrams tends to decrease. In some cases, it varied from the $100 \%$ of total incomparabilities of original HD, to only the $0-10 \%$ for the smallest $t^{*}$ values. In addition, in this work we showed how, when an appropriate set of $t^{*}$ values is chosen, a family of weighted regularized Hasse matrices can be obtained, from which one can gather statistics insights about data structure and object ordering. Finally, the effects of weighting schemes on the outcome were analysed and discusses. The proposed weighting approach resulted to effectively re-shape the final Hasse diagrams and to be robust to small weights variations.

\section{Appendix A. Supplementary data}

Data sets along with calculated $\mathbf{T}^{\mathrm{W}}$ matrices, $\mathbf{H}^{\mathrm{R}}$ families, rankings, and PCA are provided as Supplementary Material. Supplementary data associated with this article can be found, in the online version, at http://dx.doi.org/10.1016/j.chemolab.2015.08.006.

\section{References}

[1] H. Hasse, J. Martinet, Uber die Klassenzahl abelscher Zahlkörper, vol. 1, Citeseer, 1985.

[2] E. Triantaphyllou, Multi-Criteria Decision Making Methods: A Comparative Study, Springer Science \& Business Media, 2013.

[3] H.-J. Zimmermann, L. Gutsche, Multi-criteria analyse, Springer, 1991.

[4] A.H. Copeland, "A reasonable social welfare function", in University of Michigan Seminar on Applications of Mathematics to the social sciences, 1951.

[5] R. Todeschini, F. Grisoni, S. Nembri, Weighted power-weakness ratio for multicriteria decision making, Chemom. Intell. Lab. Syst. 146 (Aug. 2015) 329-336.

[6] R. Brüggemann, L. Carlsen, Partial order in environmental sciences and chemistry, Springer, 2006.

[7] R. Sánchez, R. Grau, E. Morgado, Genetic code Boolean algebras, WSEAS Trans. Biol. Biomed. 1 (2) (2004) 190-197.

[8] M. Crampes, J. de Oliveira-Kumar, S. Ranwez, J. Villerd, Visualizing social photos on a hasse diagram for eliciting relations and indexing new photos, IEEE Trans. Vis. Comput. Graph. 15 (6) (Nov. 2009) 985-992.

[9] M. Fattore, Hasse diagrams, POSET theory and fuzzy poverty measures, Riv. Internazionale Sci. Sociali 116 (1) (Jan. 2008) 63-75.

[10] R. Wieland, R. Bruggemann, Hasse diagram technique and Monte Carlo simulations, Match Commun. Math. Comput. Chem. 70 (2013) 45-59.

[11] B. Van de Walle, B. De Baets, E.E. Kerre, Fuzzy multi-criteria analysis of cutting techniques in a nuclear reactor dismantling project, Fuzzy Sets Syst. 74 (1) (Aug. 1995) 115-126.

[12] R. Bruggemann, A. Kerber, and G. Restrepo, "Ranking Objects Using Fuzzy Orders, with an Application to Refrigerants.

[13] R. Brüggemann, G. Restrepo, K. Voigt, P. Annoni, Weighting intervals and ranking, exemplified by leaching potential of pesticides, Match Commun. Math. Comput. Chem. 69 (2) (2013) 413-432.

[14] K. Voigt, R. Brüggemann, Water contamination with pharmaceuticals: data availability and evaluation approach with Hasse diagram technique and METEOR, Match Commun. Math. Comput. Chem. 54 (3) (2005) 671-689.

[16] M. Pavan, R. Todeschini, Chapter 2 Total-Order Ranking Methods, in: M. P., R. Todeschini (Eds.), Scientific Data Ranking Methods Theory and Applications, vol. 27, Elsevier 2008, pp. 51-72.

[17] P. Labadie, M. Chevreuil, Partitioning behaviour of perfluorinated alkyl contaminants between water, sediment and fish in the Orge River (nearby Paris, France), Environ. Pollut. 159 (2) (Feb. 2011) 391-397.

[18] M. Böhm, J. Stürzebecher, G. Klebe, Three-dimensional quantitative structure-activity relationship analyses using comparative molecular field analysis and comparative molecular similarity indices analysis to elucidate selectivity differences of inhibitors binding to trypsin, thrombin, and factor Xa, J. Med. Chem. 42 (3) (Feb. 1999) 458-477. 
[19] L. Carlsen, A combined QSAR and partial order ranking approach to risk assessment, SAR QSAR Environ. Res. 17 (2) (Apr. 2006) 133-146.

[20] European Communities, Public Data on High Volume Chemicals, EUR 19559EN, 2000.

[21] M. Schreiber, Twenty Hirsch index variants and other indicators giving more or less preference to highly cited papers, Ann. Phys. 522 (8) (Aug. 2010) 536-554.

[22] SCImago Journal Rank, http://www.scimagojr.com/journalrank.php?category= 1602 Jun 012015.
[23] R. Todeschini, D. Ballabio, M. Cassotti, V. Consonni, N3 and BNN: two new similarity based classification methods. An extended comparison with other classifiers, J. Chem. Inf. Model. (2015) (submitted for publication).

[24] Data Set Library, Online Available: http://support.minitab.com/en-us/datasets/ Jun 252015 Accessed:

[25] M.G. Kendall, B.B. Smith, The problem of m rankings, Ann. Math. Stat. 10 (3) (Sep. 1939) 275-287. 